\title{
Investigation of Structurally and Aerodynamically Mistuned Oscillating Cascade Using Fully-Coupled Method
}

\author{
H. M. Phan ${ }^{1}$ \\ Department of Engineering Science, University of Oxford, Oxford, United Kingdom \\ (hien.phan@eng.ox.ac.uk)
}

L. He

Department of Engineering Science, University of Oxford, Oxford, United Kingdom

\begin{abstract}
There seems to be a lack of clear and systematic understanding of physical behaviour and mechanisms of mistuned bladerows, particularly in the context of the aerodynamic mistuning versus the structural (frequency) mistuning. A high-fidelity fully-coupled method is desirable to investigate the vibration characteristics of aeroelasticity problems with strong fluid-structure interaction effects, as well as blade mistuning effects. In the present work, the direct nonlinear time-domain fully-coupled method is adopted to investigate the dynamics mechanism of a mistuned oscillating cascade. The main objectives are two-folds, firstly to elucidate the basic vibration characteristics of a mistuned bladerow, and secondly to examine the aeroelastic effects of mistuning. Three conditions of interest are considered: a) the structural mistuning only, b) the aerodynamic mistuning only, and c) a combination of the two. The present results show that firstly a mistuned configuration tends to vibrate with the same frequency and a predominantly constant inter-blade phase-angle. Vibration amplitudes of the blades vary significantly with a strong mode localization effect for the structural mistuning. For the concurrent structural-aerodynamic mistuning, the localization is stronger than in the standalone structural mistuning case. Secondly, a monotonic increase of the aeroelastic stability with the structural mistuning magnitude is observed. On the other hand, the aerodynamically mistuned cascade shows a stabilizing effect with a small amount of mistuning but exhibits a destabilizing effect with
\end{abstract}

${ }^{1}$ Corresponding author

Phan, GTP-21-1518 
ASME Journal of Engineering for Gas Turbines and Power

a large mistuning. Furthermore, the present results indicate a quite remarkable interplay between the structural and the aerodynamic mistuning. At a condition where the tuned cascade is still stable, an aeromistuning induced unstable behaviour is observed. An introduction of a large magnitude of frequency mistuning which would be stabilising for a tuned cascade, is instead shown to have a destabilizing effect for the present combined aero-structural mistuning case.

\section{INTRODUCTION}

The development of an efficient and reliable gas turbine has been continually pursued by both the aviation and power generation industries. During the development campaign, timing is critical among other essential factors. As of today, it is the industry standard to utilize the phase-shifted single-passage approach such as Linear/Nonlinear-Harmonic [1, 2] and Harmonic Balance [3] methods to investigate the flutter and forced response characteristics of potential blading design candidates. These state-of-the-art simulation methods are highly efficient and accurate thanks to two main assumptions that have been made. In the first assumption, the blades are assumed to have high inertia and stiffness such that the blade's structural responses affect marginally their aerodynamic characteristics. Thus, the aeroelastic phenomena can be studied in the decoupled manner, i.e. the fluid and the structure side can be studied separately. In this conventional approach, the modeshape and the eigen-frequency of the mode of interest are obtained from a pre-stressed modal analysis using the Finite Element approach. The blades are forced to vibrate according to a prescribed modeshape and inter-blade phase angle to compute the aerodynamic damping. For forced response problems, a modal force is obtained in a separate calculation, where the blades are stationary but the disturbances

Phan, GTP-21-1518 
are rotating. In the second assumption, the blades are assumed to be identical, which would vibrate in a monochromatic travelling wave mode [4]. Hence, the whole-annulus domain can be reduced to a phase-shifted single-passage solution.

The first assumption is widely accepted in the community since turbomachinery blades are usually made of titanium/nickel-based alloys, which are known for their excellent stiffness-to-weight ratio. However, modern gas turbine architecture has the tendency to make more use of the composite material [5]. The low mass ratio configuration has been reported to cause unusual aeroelastic phenomena in the literature due to the stronger coupling between the fluid flow and the structural dynamics. Sadeghi and Liu [6] found a significant difference of the flutter boundary predicted by the decoupled and the fully-coupled methods for a low mass ratio configuration at the subsonic flow condition. At the transonic flow condition, they reported that the blades may oscillate alternatingly around the nominal stagger angle due to alternating choked and unchoked flow in neighbouring passages. Similar nonlinear flutter phenomenon was also reported in prior publications by Carstens et al. $[7,8]$. Chahine et al. [9] studied the transonic fan rotor at various combinations of mass ratio and stiffness using both decoupled and fully-coupled methods. They found that these two methods are in good agreement over a large range of structural condition. However, for a representative composite fan blade with low mass ratio and low stiffness, the prediction discrepancy between these methods can be as high as $27.3 \%$.

On the second assumption of identical blades, this can never be achieved in practice since the blade-to-blade variations are inevitably present due to manufacturing 
tolerances, assemble processes as well as wear and tear. This problem has long been recognised by the community and is still an active subject of research. The blade-to-blade variation is known as mistuning to distinguish from the tuned blade ideal scenario. Mistuning can be classified into two main categories: structural mistuning and aerodynamic mistuning. Structural mistuning involves the change in structural parameters such as the blade mass and the blade stiffness, which in turn induces the change in the blade eigen-frequency. Sadeghi and Liu [10] found that frequency mistuning would stabilize a cascade thanks to the temporally changing phase differences between adjacent blades using the fully-coupled method for a four-blade cascade configuration. In addition, they concluded that the effectiveness of frequency mistuning depends on the strength of fluid-structure coupling. Zhai et al. [11] investigated the aeroelastic stability of a transonic compressor blade using a component-mode-based reduced order model. They reported that alternating frequency mistuning is not only robust in flutter alleviation but also potentially beneficial in the forced response relatively to the random mistuning. Utilizing the same reduced order model, Choi et al. [12] performed an optimization study to search for the most robust intentional mistuning pattern for bladed disks. They concluded that the optimized pattern might be irregular although only two blade variations $A$ and $B$ are considered in the study. Interestingly, the optimized pattern always showed a dramatic reduction of amplitude magnification due to an unintentional mistuning compared to the tuned cascade. For a more complete review of frequency mistuning, one can refer to Ref. [13]. 
On the other hand, aerodynamic mistuning involves variations in the blade stagger angle, leading and trailing edge shape, blade thickness, and camber. The early study from Hoyniak and Fleeter [14] showed that the alternating circumferential spacing mistuning enhance the stability for cases with the elastic axis at or forward of the airfoil mid-chord. When the elastic axis moved to $60 \%$ chord, the stability enhancement was minimal. Sladojecvic et al. [15] used a whole-annulus time-domain solver to investigate the effects of alternating stagger angle on the stability of fan blade. They found that small changes of stagger angle $(0.5 \mathrm{deg})$ did not have a significant effect, whereas a large value of misstagger (2deg) could be considerably destabilizing. Ekici et al. [16] introduced an approach to study aerodynamic mistuning using harmonic balance method to populate the aerodynamic coefficient matrix, which would then be used to solve the eigenvalue problem. Due to the nature of this approach, it is restricted to a set of symmetry group. Nevertheless, it was proved to be efficient in the investigation of alternating aerodynamic mistuning. They showed that the alternating blade spacing enhanced the blade stability, while the alternating blade staggering could be either beneficial or detrimental depending on the direction of mis-staggering. Ekici et al. [17] expanded the aforementioned approach to investigate the problem of leading edge blending. They reported that alternating blending would be stabilizing for a loaded rotor but destabilizing for an unloaded rotor. Miyakozawa et al. [18] studied the effects of aerodynamic asymmetry on forced response by perturbing the influence coefficient matrix in the physical coordinates. The results showed that the amplification factor increased as the strength of random force perturbation increases. However, aerodynamic forcing perturbation had a 
secondary effect compared to frequency mistuning. In a recent publication by the authors of the present work [19], an efficient method based on the multi-scale approach was proposed to investigate the effects of random mis-staggering. It was shown that misstaggering within only \pm 1 deg from the nominal stagger angle can change the timeaveraged blade loading by up to $20 \%$. Dynamic response of the blade due to aerodynamic forcing was also observed to change significantly from the tuned cascade. The results obtained using the multi-scale method was validated against the direct simulation results, revealing a comparable accuracy but with a much reduced computational cost. Leng and Key $[20,21]$ built a method based on a generalized flat plate cascade model to investigate non-uniform blade spacing effects. They reported that the sinusoidal spacing pattern had the largest blade-to-blade variation, in which some blades experienced an unsteady loading three times higher than the tuned blade. Regarding the aerodynamic damping, they found that some mistuned blades could even have negative aero-damping at certain excitation nodal diameters, while the tuned bladerow was stable. Malzacher et al. [22] performed the mis-staggering experimental investigation on a low-speed linear cascade. They concluded that mis-staggering could either have a beneficial or detrimental effect depending on the mistuning strength. However, the cascade stability seemed not to be affected significantly by the aerodynamic mistuning.

With the background as introduced so far, one can see that fluid-structure interaction can induce significant effects on the blade aeroelastic response. Under certain circumstances, the coupling effect is prominent such that non-conventional aeroelastic phenomena can arise, which we would otherwise not be able to capture using a 
decoupled method. Regarding the mistuning effects, structural mistuning has been studied more extensively in the past compared to aerodynamic mistuning, although the latter receives more attention recently. Nevertheless, numerical investigations of mistuning typically use some types of reduced-order methods to overcome the high computational cost. Thus, it is important to investigate the basic mistuning mechanisms using fully-coupled methods, which has been a rare practice in the past. In the present work, we'll first validate the fully-coupled solution against the decoupled solution for an oscillating compressor cascade at a high mass ratio condition. Then, the multi-passage configurations with a random mistuning will be investigated to assess the vibration characteristics of structural mistuning as well as aerodynamic mistuning. The aeroelastic impacts of an alternating and a random mistuning pattern will be studied to aid the understanding of mistuning mechanisms.

\section{NUMERICAL METHODS}

Fully-coupled method involves the two-way interaction between the fluid part and the structure part. There are two time step loop: the outer loop and the inner loop. The outer loop represents the physical timestep, while the inner loop represents the pseudotimestep. At each inner loop iteration, data are exchanged between the fluid and the structure solvers. The structure solver receives the information of the aerodynamic forces acting on the blade, while the fluid solver receives the updated blade position. The iterations continue until reaching the convergence criteria. The coupling level depends on the frequency of data exchange. In the tightly coupled simulation process, data exchange 
occurs every inner loop iteration. On the other hand, if the loosely coupled simulation method is adopted, data exchange may take place at every outer loop iteration. Depending on the strength of fluid-structure interaction effects, a suitable coupling level can be used. In the present work, the tightly coupled method is adopted at the expense of computational resources. Because the tightly coupled fluid-structure interaction method is two-way iteratively implicit, the vibration characteristics is part of the solution. Thus, the method is suitable for an investigation of the unknown a-priori vibration characteristics as in the present work with the mistuned oscillating cascade.

On the fluid side, the unsteady Navier-Stokes equations are solved iteratively in an implicit manner:

$$
\begin{gathered}
\frac{\partial \rho}{\partial t}+\nabla \cdot(\rho \boldsymbol{U})=0 \\
\frac{\partial(\rho \boldsymbol{U})}{\partial t}+\nabla \cdot(\rho \boldsymbol{U} \otimes \boldsymbol{U})=-\nabla p+\nabla \cdot \tau \\
\frac{\partial(\rho H)}{\partial t}-\frac{\partial p}{\partial t}+\nabla \cdot(\rho \boldsymbol{U} H)=\nabla \cdot(\lambda \nabla T)+\nabla \cdot(\boldsymbol{U} \cdot \tau)
\end{gathered}
$$

The governing equations represent the conservation of mass, momentum, and energy at each instantaneous time level. To close the equations, the $\gamma-\theta$ transition correlation coupled with the Shear Stress Transport (SST) model is adopted [23].

On the solid side, the rigid body equations of motion are solved separately for the torsion mode:

$$
\frac{d \Pi}{\partial t}=M_{\text {aero }}-k_{\text {torsion }}\left(\theta-\theta_{\text {COM }}\right)
$$


where $\Pi$ is angular momentum. $M_{\text {aero }}$ denotes the aerodynamic moment acting on the blade. $k_{\text {torsion }}$ represents the torsional stiffness.

For a decoupled simulation, a mode shape with fixed oscillation amplitude is used to drive the blade movement. The mesh motion is therefore known a-priori. At each time instant, the mesh motion equation will be solved separately from the flow equations, thus it is known as a decoupled simulation. On the other hand, for a fully-coupled simulation, the instantaneous oscillation amplitude is not known a-priori. It is an implicit result from the two-way balance of flow and rigid body equations such that the dependencies between the fluid and the body are converged within a time step. Within the fully-coupled framework, there are more than one way to initialize/start the simulation. For turbomachinery applications, it is a common practice to impose a specific initial interblade phase angle pattern according to the investigated nodal diameter [24-26]. This practice stems from the monochromatic travelling wave behaviour of turbomachinery flutter [4]. On the other hand, only few researchers used a non-travelling wave perturbation as an initial condition $[7,27]$ due to a high number of oscillation cycles needed to establish the coupled vibration characteristics, particularly for aerodynamically induced instabilities [28]. Although a travelling wave perturbation is more efficient, a nontravelling wave initialization is necessary if the vibration characteristics are not known a priori. In the present work, vibration characteristics of the mistuned cascade are of interest. Thus, single-blade perturbation will be adopted for mistuned configuration, unless otherwise stated. A travelling wave perturbation will only be used for comparison between the decoupled and fully-coupled solutions. 


\section{TEST CASE DESCRIPTION AND VALIDATIONS}

\subsection{Test Case Description}

The blade profile adopted in the present paper is of control-diffusion type, which is the representative of modern blading designs. Since the work of Elazar and Shreeve [29], this blade profile has been studied extensively both experimentally and numerically. The experimental results presented in the current study for validation purposes are acquired from the oscillating cascade test [30]. The experiment was conducted at a reduced Reynolds number $R e=1.95 \times 10^{5}$ compared to the original design Reynolds number $R e=7 \times 10^{5}$.

Table 1 shows a comparison between experimental and simulated operating conditions. Reynolds number $R e$ and exit isentropic velocity $V_{r e f}$ are matched to a less than $5 \%$ discrepancy from the experimental conditions.

Regarding the modeshape, only the torsional vibration mode will be investigated for mistuned configurations. The torsional axis is located at the blade mid-chord.

Some essential non-dimensional parameters governing the vibration characteristics include the mass ratio $\mu$ and the reduced frequency $k$. The former is the ratio between the blade mass and the mass of surrounding control-volume fluid, whilst the latter dictates the level of unsteadiness:

$$
\mu=\frac{m_{\text {blade }}}{\rho \pi(C / 2)^{2} h}
$$

where $C$ is the blade chord and $h$ is the blade height, and 
ASME Journal of Engineering for Gas Turbines and Power

$$
k=\frac{\omega C}{V_{r e f}}
$$

where $V_{r e f}$ is the reference isentropic velocity.

\subsection{Validations}

Firstly, the steady flow characteristics of the blade profile are assessed. Fig. 1 presents the steady pressure distribution at mid-span. The computational predictions agree well with the experimental results. A separation bubble formation around 40 - $60 \%$ chord can be observed in the experiment. Interestingly, this separation bubble can only be captured in the computation if the transitional turbulence model is used [31]. Instead, a rather smooth pressure distribution at this region is captured with the fully turbulent model.

Next, the overall damping of the tuned oscillating cascade in the bending mode are validated against the experiment as shown in Fig. 2. Overall, the influence coefficient method computational results agree well with the experimental measurement. The most unstable inter-blade phase angle has been predicted fairly well at IBPA about 20deg to $30 \mathrm{deg}$ for both reduced frequencies. For more details and analyses for the validation of the tuned oscillating cascade in the bending mode, particularly for the effects of laminar separation bubble and tip clearance, one is referred to Ref. [31].

For validation purposes against the decoupled method, a high mass ratio $\mu=800$ is adopted to improve the quasi-periodic vibration of the fully-coupled cascade. Before studying the mistuning effects, the fully-coupled solution of the torsional vibration is validated against the uncoupled solution for the tuned cascade. These numerical methods 
should be in good agreement with each other for a high mass ratio configuration, reflecting the suitability of the industry-standard uncoupled solution at a relatively small fluid-structure interaction effect. Fig. 3 compares the overall damping curve computed by the uncoupled and fully-coupled methods for the torsional vibration mode. Among the uncoupled solutions, there is an excellent agreement between the influence coefficient method (ICM) and the travelling-wave tuned cascade across the entire range of IBPAs. The log-dec is calculated by the fully-coupled method from the successive peaks, whereas the aerodynamic damping calculated from the energy method is converted to log-dec for the uncoupled method. Across an investigated range of IBPAs, the uncoupled and fully coupled methods are well-matched. We note that the local discrepancies between the uncoupled and fully-coupled methods stem from the changing (i.e. decaying or amplifying) vibration amplitude of the fully-coupled solution in contrast to the constant vibration amplitude in the uncoupled method, which is related to the log-dec value. Thus, the local responses at IBPA $\sigma=0^{\circ}$ (minimum log-dec) and IBPA $\sigma=-120^{\circ}$ (maximum log-dec) will be further examined.

Fig. 4 presents the unsteady pressure responses at IBPA $\sigma=0^{0}$, whereas Fig. 5 presents that at IBPA $\sigma=-120^{\circ}$. An excellent agreement between the predictions by uncoupled and coupled methods is observed at $\sigma=0^{0}$. Regarding the case of $\sigma=$ $-120^{\circ}$, a general trend is captured by both uncoupled and fully-coupled methods. However, there are still some local discrepancies, such as around $40-60 \%$ chord on the suction surface and around $20-40 \%$ chord on the pressure surface. At mid-chord on the blade suction surface, there is a laminar separation bubble, followed by the transition to 
turbulence. From $20 \%$ to $40 \%$ chord on the blade pressure surface, a laminar separation bubble is formed although no subsequent transition has been observed. At these separated regions, the predictions by the uncoupled and fully-coupled deviate locally from each other. However, these local discrepancies are confined to a rather small area thus its global damping are not significantly affected.

\section{BASIC VIBRATION CHARACTERISTICS OF MISTUNED CASCADE}

\subsection{Random Structural Mistuning}

In this study, structural mistuning is realized by changing the blade stiffness while retaining its mass and inertia, thus effectively changes the eigen-frequency of each individual blade. Hence, this type of structural mistuning is also known as frequency mistuning. The random mistuning pattern is illustrated in Fig. 6, where the mistuned frequency is within the range of $\pm 1 \%$ from the nominal tuned cascade.

Fig. 7 compares the eigen-frequency and oscillating frequency of the randomly mistuned cascade. The oscillating frequency is obtained for each individual mistuned blade by performing Fast Fourier transform (FFT) on its vibration history. It is interesting to note that all mistuned blades eventually couple and oscillate at the same frequency although each blade has a rather different eigen-frequency to start with.

Fig. 8 presents the phase evolution of each blade with time. It can be seen that the vibrations of all blades follow a horizontal line (denoted as a white dashed line in Fig. 8), which mean that all blades are largely in phase with each other. This represents a standing wave with a constant IBPA $\sigma=0^{0}$.

Phan, GTP-21-1518 
Fig. 9 presents the normalized blade vibration amplitude against the cascade-average value during a specific oscillation cycle. If the tuned cascade theory still holds [4], one would expect to observe a constant vibration amplitude across the cascade. Instead, vibration amplitudes can be seen to vary among blades for the structurally mistuned configuration. The variation pattern can be related to the imposed structural mistuning pattern (see Fig. 6), in which high eigen-frequency blades tends to have high vibration amplitudes. The magnitude of variation is also large with up to $\pm 40 \%$ difference compared to the mean value of the whole bladerow.

\subsection{Random Aerodynamic Mistuning}

In the present work, aerodynamic mistuning is realized by changing the blade stagger angle. A twelve-blade randomly mistuned cascade is reused with the same mistuning pattern as in the previous case. However, this time each blade has the same eigenfrequency but different (mean) stagger angle. The random mis-stagger angle is within the range of \pm 1 deg from the nominal conditions. Fig. 10 illustrates the convention of negative/positive mis-staggered blade in the aerodynamically mistuned cascade.

Fig. 11 presents the imposed steady mis-staggered pattern and the mean vibration position. The mis-staggering pattern is randomly distributed within the range of $\pm 0.5 \mathrm{deg}$ from the tuned nominal position. The mean vibration positions can be seen to be consistent with the steady mis-staggered pattern. This suggests that the unsteady interactions of the aero-mistuned blades in this particular test case and condition have small influence on the time-averaged (steady) responses. 
All blades in the aerodynamically mistuned cascade have the same structural properties, thus the same eigen-frequency. Therefore, it is not surprising that all blades oscillate at the same coupled frequency. We then turn our attention to the phase angle distribution of the aerodynamically mistuned cascade as shown in Fig. 12. Similarly to the tuned cascade, the inter-blade phase angle remains constant across the cascade. In this case, a standing-wave $\left(\sigma=0^{0}\right)$ is observed as denoted by the white dashed line.

Fig. 13 presents the normalized vibration amplitude of the aero-mistuned cascade in comparison with the expectation from the tuned cascade. Vibration amplitudes can be seen to vary among blades, although the variation ( $\pm 15 \%)$ is much less than that of the structurally mistuned configuration ( $\pm 40 \%)$.

\subsection{Concurrent Structural-Aerodynamic Mistuning}

In reality, both structural and aerodynamic mistuning will be inevitably co-existing in a bladerow. A standalone structural or aerodynamic mistuning is still a simplified representation of the mistuned system, although it has been useful to investigate its effect in a separate manner. In this section, we attempt to study the concurrent structural-aerodynamic mistuned bladerow. The mistuned eigen-frequency distribution is the same as in the standalone structural mistuning case, while the mis-staggered angle distribution is the same as in the standalone aerodynamic mistuning case. From Fig. 14, the mean vibration positions of the concurrent structural-aero mistuning resemble that of the aero mistuning only configuration. This suggests aero-mistuning is solely responsible for the mis-staggering characteristics.

Phan, GTP-21-1518 
Fig. 15 shows the frequency distribution of the concurrent structural-aero mistuned cascade. Similarly to the case with structural mistuning only, the coupled oscillating frequency is constant across the cascade regardless of the initial randomly distributed eigen-frequency.

Fig. 16 tracks the instantaneous phase temporal evolution of each blade across the concurrent structural-aero mistuned cascade. Vibrations of all blades follow a horizontal line (denoted as a white dashed line in Fig. 16), which mean that all blades are largely in phase with each other. This represents a standing wave with a constant IBPA $\sigma=0^{0}$.

Fig. 17 presents the normalized vibration amplitude of the concurrent structural-aero mistuned cascade in comparison with the standalone structural mistuning. Vibration amplitudes can be seen to vary among blades with the variation magnitude up to $\pm 60 \%$. This variation magnitude is higher than both standalone structural or aerodynamic mistuning, which suggests that structural mistuning interacts with aerodynamic mistuning to amplify the mode localization effect.

\section{AEROELASTIC IMPACTS OF MISTUNING}

\subsection{Alternating Mistuning}

\subsubsection{Standalone Structural Mistuning}

In this section, we will confine our investigations on the aeroelastic stability of an alternating mistuned cascade. The alternating mistuned pattern is illustrated in Fig. 18, where the odd-numbered blades have higher eigen-frequency and even-numbered blades have lower eigen-frequency. The mean eigen-frequency of the mistuned cascade 
is the same of that in the tuned cascade. Four mistuning amplitudes will be studied, namely $1 \%, 2 \%, 3 \%$, and $4 \%$. Small values of mistuning strength is inevitably present in real configurations, while larger mistuning can be regarded as an intentionally mistuned design.

Fig. 19 compares the normalized log-dec of an alternating structurally mistuned cascade with a range of mistuning strength and mass ratio. Structural mistuning is observed to be stabilizing for all investigated cases. The stabilization effect increases monotonically as the mistuning strength increases. In addition, the mistuned cascade with higher mass ratio shows stronger stabilization effect.

It was observed in the first part of the present work that the vibration amplitudes in a structurally mistuned cascade would be scattered. This is also known as the localized vibration phenomena. Fig. 20 presents the modal amplitude ratio for a range of mistuning strength and blade mass ratio. The modal amplitude ratio is the proportion of vibration amplitudes of the odd-numbered blade to that of the even-numbered blades in the alternating mistuned cascade. It can be seen that the modal amplitude ratio increases with the mistuning strength. The amount and rate of modal amplitude ratio increase is more obvious as the mass ratio rises. The trend of modal amplitude ratio (Fig. 20) is similar to that of log-dec (Fig. 19), thus suggesting that the gain in log-dec is associated with the stronger localization effect. For further analysis on the relationship between the mode localization and aeroelastic stability improvement, one is referred to Ref. [32]. 


\subsubsection{Standalone Aerodynamic Mistuning}

Next, the alternating aerodynamic mistuned cascade is investigated. The oddnumbered blades are positively mis-staggered, while the even-numbered blades are negatively mis-staggered. The mean stagger angle of the mistuned cascade is the same of that in the tuned cascade. Three mis-staggered angles will be studied, namely 0.5, 1.0, and 1.5 degrees. Fig. 21 compares the normalized log-dec of an alternating aerodynamic mistuned cascade with a range of mistuning strength and mass ratio. Interestingly, logdec first increases for small values of mis-staggered angle then decreases as the misstaggered amplitudes become bigger. This tendency is affected marginally by the blade mass ratio. There was an attempt to link the change of aeroelastic stability in an aeromistuned cascade with its unsteady aerodynamic characteristics to explain the nonmonotonic behaviour. One is referred to Ref. [32] for further analysis regarding this aspect.

Although the aero-mistuned cascade is more destabilizing at high values of misstaggering compared to the tuned cascade (see Fig. 21), the aero-mistuned cascade is still stable without any sign of flutter at the high reduced frequency condition $k=0.8$. It would be of interest to explore and answer a question: if a blade row is structurally tuned, can there be an operating condition at which the aero-mistuned cascade is unstable but the aero-tuned cascade is stable?

To answer the above research question, we lower the reduced frequency to $k=0.3$ to promote the flutter susceptibility. Interestingly, a contrasting behaviour between the fully tuned cascade and the alternating aero-mistuned but structurally tuned cascade can be

Phan, GTP-21-1518 
observed as shown in Fig. 22. Although the fully tuned cascade's vibration is decaying (thus stable, as shown in Fig. 22a), the vibratory displacement of the alternating aeromistuned but structurally-tuned cascade amplifies with time, i.e. unstable (Fig. 22b).

\subsubsection{Concurrent Structural-Aerodynamic Mistuning}

Given that the standalone structural mistuning has a stabilizing effect as observed in the previous section, another different but related research question arises: what is the interaction between an aerodynamic mistuning and a structural mistuning, when they copresent in the cascade? Particularly for the already unstable alternating aero-mistuned configuration, the impacts of additional structural mistuning would be of interest.

Fig. 23 presents the amplitude evolution for the aero-mistuned cascade subject to additional 1\% structural mistuning (Fig. 23a) and 4\% structural mistuning (Fig. 23b). It can be seen that both configurations still experience flutter with amplifying vibration amplitudes. It is reminded that $4 \%$ structural mistuning by itself could increase five-fold the aeroelastic stability at $\mathrm{k}=0.8$ (see Fig. 19) compared to the tuned cascade. However, the additional alternating structural mistuning in the present unstable aero-mistuned cascade is shown to behave differently. Quite remarkably, the additional structural mistuning seems to result in an opposite adverse effect now. The seemingly destabilizing effect of the structural mistuning manifests in terms of a strong localization which exacerbates the instability of the most unstable blade. As the structural mistuning increases from $1 \%$ to $4 \%$, the most unstable blade reaching a larger amplitude after a fewer number of oscillation cycles.

Phan, GTP-21-1518 


\subsection{Random Mistuning}

The previous work has been carried out with an alternating mistuning configuration. It is imperative to check whether the mistuning pattern would affect our findings. In this section, we use an eight-passage configuration subject to random mistuning pattern.

\subsubsection{Standalone Aerodynamic Mistuning}

Fig. 24a shows that the eight-passage tuned cascade is stable with decaying amplitude, in agreement with the previous finding. This suggests that the final vibration mode of the tuned cascade is not sensitive to the computational domain size. On the other hand, Fig. 24b shows that the randomly aero-mistuned configuration does not experience flutter unlike in the alternating mistuning case. Nevertheless, the fluctuating amplitudes in Fig. 24b point out that the vibration has a beating characteristics that stands out from the tuned configuration.

\subsubsection{Concurrent Structural-Aerodynamic Mistuning}

A random structural mistuning is further added to the randomly aero-mistuned cascade. Fig. 25 shows the amplitude evolution of the aero-mistuned cascade with additional 1\% structural mistuning (Fig. 25a) and 4\% structural mistuning (Fig. 25b). Again, it is clear that the additional structural mistuning has the possibility to exacerbate the cascade instability. With $4 \%$ structural mistuning, the most vulnerable blade in the cascade reaches a larger vibration amplitude. As a result, it can be concluded that adding

Phan, GTP-21-1518 
the structural mistuning to the pure aero-mistuned cascade does not always alleviate the instability. On the contrary the added structural mistuning can amplify the vibration amplitude of the most vulnerable blade, most likely due to the associated strong modal localization.

\section{CONCLUSIONS}

The objectives of the present paper are to enhance the fundamental understanding of the vibration characteristics and the aeroelastic impacts of the structurally and aerodynamically mistuned cascade. The high-fidelity nonlinear time-domain fully-couple method has been used, without any frequency-domain related CFD reduced-order modeling assumptions.

Regarding the basic vibration characteristics of the mistuned cascade, the main observations are:

- Standalone structural mistuning: blades vibrate with the same coupled frequency, a predominantly constant inter-blade phase angle, but non-uniform amplitudes with a strong amplitude localization.

- Standalone aerodynamic mistuning: blades vibrate with the same coupled frequency, a constant inter-blade phase angle, a weak amplitude localization, and varying mean blade positions.

- Concurrent structural-aerodynamic mistuning: blades vibrate with the same coupled frequency, a predominantly constant inter-blade phase angle. The amplitude localization seems stronger than the standalone structural mistuning

Phan, GTP-21-1518 
case. The mean positions are similar to the standalone aerodynamic mistuning case.

Regarding the aeroelastic impact of mistuning in the second part of the work, the following observations are made:

- Standalone structural mistuning: it always has a stabilizing effect. The degree of stabilization strongly correlates to that of the aeroelastic coupling in terms of the blade mass coefficient.

- Standalone aerodynamic mistuning: it has a stabilizing effect with a small amount of mistuning but shows a destabilizing effect with a large mistuning. An aeromistuning induced flutter with an alternating configuration has been reported for the first time. It shows that an aerodynamic mistuning can lead to flutter at a condition where the tuned cascade is still stable.

- Concurrent structural-aerodynamic mistuning: when both aerodynamic and structural mistuning are present, there seems to be a distinctive nonlinear interaction between the two. It has been shown that the stability of the unstable aero-mistuned cascade may be improved with a small additional structural mistuning, but degraded remarkably with a larger value of additional structural mistuning. 
ASME Journal of Engineering for Gas Turbines and Power

\section{ACKNOWLEDGMENT}

The support from the EPSRC CDT in Gas Turbine Aerodynamics is much appreciated. The work is partly sponsored by the Chair of Computational Aerothermal Engineering Bursary. The authors would like to acknowledge the use of the University of Oxford Advanced Research Computing (ARC) facility in carrying out this work (http://dx.doi.org/10.5281/zenodo.22558). 


\section{NOMENCLATURE}

\begin{tabular}{|c|c|}
\hline FFT & fast Fourier transform \\
\hline IBPA & inter-blade phase angle (also $\sigma$ ), deg \\
\hline SS & suction surface \\
\hline PS & Pressure surface \\
\hline$k$ & reduced frequency; $k=\omega C / V_{\text {ref }}$ \\
\hline$C$ & blade chord, $\mathrm{m}$ \\
\hline$C_{a x}$ & blade axial chord, $\mathrm{m}$ \\
\hline$C_{p}$ & steady pressure coefficient; $C_{p}=\left(P-P_{2}\right) /\left(P_{01}-P_{2}\right)$ \\
\hline$\left|C_{p}\right|$ & $1^{\text {st }}$ harmonic unsteady pressure amplitude \\
\hline$\phi$ & $1^{\text {st }}$ harmonic unsteady pressure phase angle \\
\hline$\omega$ & angular frequency, $\mathrm{rad} \mathrm{s}^{-1}$ \\
\hline$P_{01}$ & mid-span inlet total pressure, $\mathrm{Pa}$ \\
\hline$P_{2}$ & outlet static pressure, $\mathrm{Pa}$ \\
\hline$V_{\text {ref }}$ & reference isentropic exit velocity, $\mathrm{ms}^{-1} ; V_{r e f}=\sqrt{2\left(P_{01}-P_{2}\right) / \rho}$ \\
\hline$x$ & axial distance, $\mathrm{m}$ \\
\hline$\gamma$ & stagger angle, deg \\
\hline
\end{tabular}




\section{REFERENCES}

[1] Ning, W., and He, L., 1998, "Computation of Unsteady Flows Around Oscillating Blades Using Linear and Nonlinear Harmonic Euler Methods," J. Turbomach., 120(3), pp. 508514. DOI: $10.1115 / 1.2841747$

[2] He, L., and Ning, W., 1998, "Efficient Approach for Analysis of Unsteady Viscous Flows in Turbomachines," AIAA J., 36(11), pp. 2005-2012. DOI: 10.2514/2.328

[3] Hall, K. C., Thomas, J. P., and Clark, W. S., 2002, "Computation of Unsteady Nonlinear Flows in Cascades Using a Harmonic Balance Technique," AIAA J., 40(5), pp. 879-886. DOI: $10.2514 / 2.1754$

[4] Lane, F., 1956, "System Mode Shapes in the Flutter of Compressor Blade Rows," J. Aeronaut. Sci., 23(1), pp. 54-66. DOI: 10.2514/8.3502

[5] Brandstetter, C., Duquesne, P., Paoletti, B., Aubert, S., and Ottavy, X., 2019, "Project PHARE-2 - A High Speed UHBR Fan Test Facility for a New Open-Test Case," J. Turbomach., 141(10), p. 101004. DOI: 10.1115/1.4043883

[6] Sadeghi, M., and Liu, F., 2005, "Computation of Cascade Flutter by Uncoupled and Coupled Methods," Int. J. Comput. Fluid Dyn., 19(8), pp. 559-569. DOI: 10.1080/10618560500508367

[7] Carstens, V., Kemme, R., and Schmitt, S., 2003, "Coupled Simulation of Flow-Structure Interaction in Turbomachinery," Aerosp. Sci. Technol., 7(4), pp. 298-306. DOI: 10.1016/S1270-9638(03)00016-6

[8] Carstens, V., and Belz, J., 2001, "Numerical Investigation of Nonlinear Fluid-Structure Interaction in Vibrating Compressor Blades," J. Turbomach., 123(2), pp. 402-408. DOI: 10.1115/1.1354138

[9] Chahine, C., Verstraete, T., and He, L., 2019, "A Comparative Study of Coupled and Decoupled Fan Flutter Prediction Methods Under Variation of Mass Ratio and Blade Stiffness," J. Fluids Struct., 85, pp. 110-125. DOI: 10.1016/j.jfluidstructs.2018.12.009

[10] Sadeghi, M., and Liu, F., 2007, "Investigation of Mistuning Effects on Cascade Flutter Using a Coupled Method," AIAA J. Propul. Power, 23(2), pp. 266-272. DOI: 10.2514/1.18876

[11] Zhai, Y., Bladh, R., and Dyverfeldt, G., 2012, "Aeroelastic Stability Assessment of an Industrial Compressor Blade Including Mistuning Effects," J. Turbomach., 134(6), p. 060903. DOI: 10.1115/1.4007210

[12] Choi, B. K., Lentz, J., Rivas-Guerra, A. J., and Mignolet, M. P., 2003, "Optimization of Intentional Mistuning Patterns for the Reduction of the Forced Response Effects of Unintentional Mistuning: Formulation and Assessment," ASME J. Eng. Gas Turbines Power, 125(1), pp. 131-140. DOI: 10.1115/1.1498270

[13] Castanier, M. P., and Pierre, C., 2006, "Modeling and Analysis of Mistuned Bladed Disk Vibration - Current Status and Emerging Directions," AIAA J. Propul. Power, 22(2), pp. 384-396. DOI: 10.2514/1.16345

[14] Hoyniak, D., and Fleeter, S., 1986, "The Effect of Circumferential Aerodynamic Detuning on Coupled Bending-Torsion Unstalled Supersonic Flutter," J. Turbomach., 108(2), pp. 253-260. DOI: 10.1115/1.3262045 
[15] Sladojevic, I., Sayma, A. I., and Imregun, M., 2007, "Influence of Stagger Angle Variation on Aerodynamic Damping and Frequency Shifts," ASME Paper No. GT200728166. DOI: 10.1115/GT2007-28166

[16] Ekici, K., Kielb, R. E., and Hall, K. C., 2010, "Aerodynamic Assymetry Analysis of Unsteady Flows in Turbomachinery," J. Turbomach., 132(1), p. 011006. DOI: 10.1115/1.3103922

[17] Ekici, K., Kielb, R. E., and Hall, K. C., 2013, "The Effect of Aerodynamic Asymmetries on Turbomachinery Flutter," J. Fluids Struct., 36, pp. 1-17. DOI: 10.1016/j.jfluidstructs.2012.08.009

[18] Miyakoyawa, T., Kielb, R. E., and Hall, K. C., 2009, "The Effects of Aerodynamic Perturbations on Forced Response of Bladed Disks," J. Turbomach., 131(4), p. 041008. DOI: $10.1115 / 1.3068319$

[19] Phan, H. M., and He, L., 2021, "Efficient Steady and Unsteady Flow Modeling for Arbitrarily Mis-Staggered Bladerow Under Influence of Inlet Distortion," ASME J. Eng. Gas Turbines Power, 143(7), p. 071009. DOI: 10.1115/1.4050364

[20] Leng, Y., and Key, N. L., 2020, "Effects of Nonuniform Blade Spacing on Compressor Rotor Forced Response and Aeroacoustics," AIAA J. Propul. Power, 36(5), pp. 721-731. DOI: 10.2514/1.B37866

[21] Leng, Y., and Key, N. L., 2021, "The Effects of Non-Uniform Blade Spacing on Compressor Rotor Flutter Stability," AIAA J. Propul. Power. DOI: 10.2514/1.B37765

[22] Malzacher, L., Schwarze, C., Motta, V., and Peitsch, D., 2019, "Experimental Investigation of an Aerodynamically Mistuned Oscillating Compressor Cascade," J. Turbomach., 141(7), p. 071012. DOI: 10.1115/1.4043474

[23] Menter, F. R., Langtry, R. B., Likki, S. R., Suzen, Y. B., Huang, P. G., and Völker, S., 2006, "A Correlation-Based Transition Model Using Local Variables - Part I: Model Formulation," ASME J. Turbomach., 128(3), pp. 413-422. DOI: 10.1115/1.2184352

[24] Doi, H., and Alonso, J. J., 2002, "Fluid/Structure Coupled Aeroelastic Computations for Transonic Flows in Turbomachinery," ASME Paper No. GT2002-30313. DOI: 10.1115/GT2002-30313

[25] Vahdati, M., Simpson, G., and Imregun, M., 2011, "Mechanisms for Wide-Chord Fan Blade Flutter," ASME J. Turbomach., 133(4), p. 041029. DOI: 10.1115/1.4001233

[26] Im, H. S., and Zha, G. C., 2012, "Simulation of Non-Synchronous Blade Vibration of an Axial Compressor Using a Fully Coupled Fluid/Structure Interaction," ASME Paper No. GT2012-68150. DOI: 10.1115/GT2012-68150

[27] Corral, R., and Gallardo, J. M., 2014, "Nonlinear Dynamics of Bladed Disks with Multiple Unstable Modes," AIAA J., 52(6), pp. 1124-1132. DOI: 10.2514/1.J051812

[28] He, L., 1994, "Integration of 2-D Fluid/Structure Coupled System for Calculations of Turbomachinery Aerodynamic/Aeroelastic Instabilities," Int. J. Comput. Fluid Dyn., 3(3-4), pp. 217-231. DOI: 10.1080/10618569408904508

[29] Elazar, Y., and Shreeve, R. P., 1990, "Viscous Flow in a Controlled Diffusion Compressor Cascade with Increasing Incidence," ASME J. Turbomach., 112(2), pp. 256-265. DOI: 10.1115/1.2927642 
[30] Yang, H., and He, L., 2004, "Experimental Study on Linear Compressor Cascade with Three-Dimensional Blade Oscillation," AIAA J. Propul. Power, 20(1), pp. 180-188. DOI: $10.2514 / 1.1280$

[31] Phan, H. M., and He, L., 2020, "Validation Studies of Linear Oscillating Compressor Cascade and Use of Influence Coefficient Method," ASME J. Turbomach., 142(5), p. 051005. DOI: 10.1115/1.4045657

[32] Phan, H. M., and He, L., 2021, "Investigation of Mistuned Oscillating Cascade Using Fully-Coupled Method," ASME Paper No. GT2021-59124 


\section{Figure Captions List}

Fig. 1 Steady pressure distribution at mid-span

Fig. 2 Overall damping for bending mode at $\mathrm{k}=0.2$ and 0.4

Fig. $3 \quad$ Overall damping for torsion mode at $\mathrm{k}=0.8$

Fig. 4 Unsteady pressure responses at IBPA $\sigma=0^{0}:$ (a) Amplitude and b) Phase angle

Fig. 5 Unsteady pressure responses at IBPA $\sigma=-120^{\circ}$ : (a) Amplitude and b) Phase angle

Fig. 6 Random mistuning pattern

Fig. $7 \quad$ Random structural mistuning frequency distribution for all blades

Fig. $8 \quad$ Random structural mistuning phase distribution for all blades

Fig. 9 Random structural mistuning amplitude distribution for all blades

Fig. 10 Aerodynamic mistuning illustration

Fig. 11 Steady/mean mis-staggering for all blades in the random aerodynamic mistuning

Fig. 12 Random aerodynamic mistuning phase distribution for all blades

Fig. 13 Random aerodynamic mistuning amplitude distribution for all blades

Fig. 14 Steady/mean mis-staggering for all blades in the concurrent structuralaerodynamic mistuning 
Fig. 15 Concurrent structural-aerodynamic mistuning frequency distribution for all blades

Fig. 16 Concurrent structural-aerodynamic mistuning phase distribution for all blades

Fig. 17 Concurrent structural-aerodynamic mistuning amplitude distribution for all blades

Fig. 18 Alternating mistuning pattern of $1 \%$

Fig. 19 Alternating structural mistuning log-dec at $k=0.8$

Fig. 20 Alternating structural mistuning amplitude ratio at $\mathrm{k}=0.8$

Fig. $21 \quad$ Alternating aerodynamic mistuning log-dec at $k=0.8$

Fig. 22 Amplitude evolution for the: (a) Aerodynamically tuned + Structurally tuned and (b) Alternating aero-mistuning $1.5 \mathrm{deg}+$ Structurally tuned cascade

Fig. 23 Amplitude evolution for the: (a) Alternating aero-mistuning $1.5 \mathrm{deg}+$ Alternating structural mistuning $1 \%$ and (b) Alternating aero-mistuning $1.5 \mathrm{deg}+$ Alternating structurally mistuning $4 \%$ cascade

Fig. 24 Amplitude evolution for the: (a) Aerodynamically tuned + Structurally tuned and (b) Random aero-mistuning 1.5deg + Structurally tuned cascade

Fig. 25 Amplitude evolution for the: (a) Random aero-mistuning 1.5deg + Random structural mistuning $1 \%$ and (b) Random aero-mistuning 1.5deg + Random structurally mistuning $4 \%$ cascade 


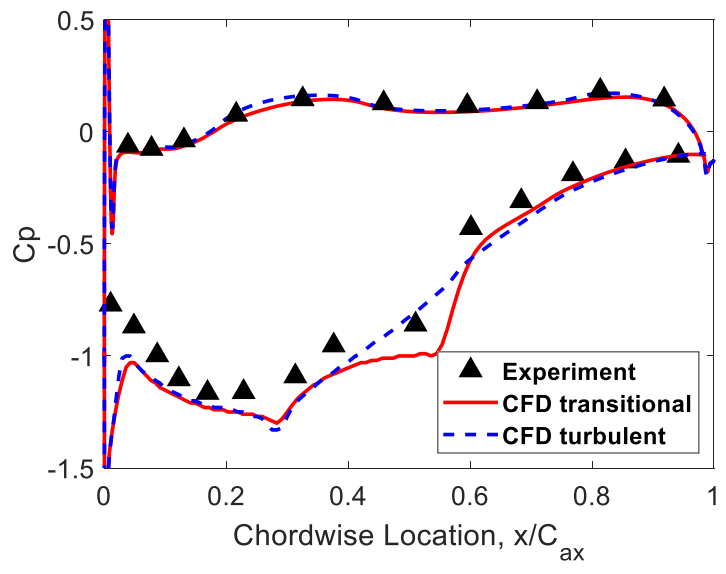

Fig. 1 Steady pressure distribution at mid-span

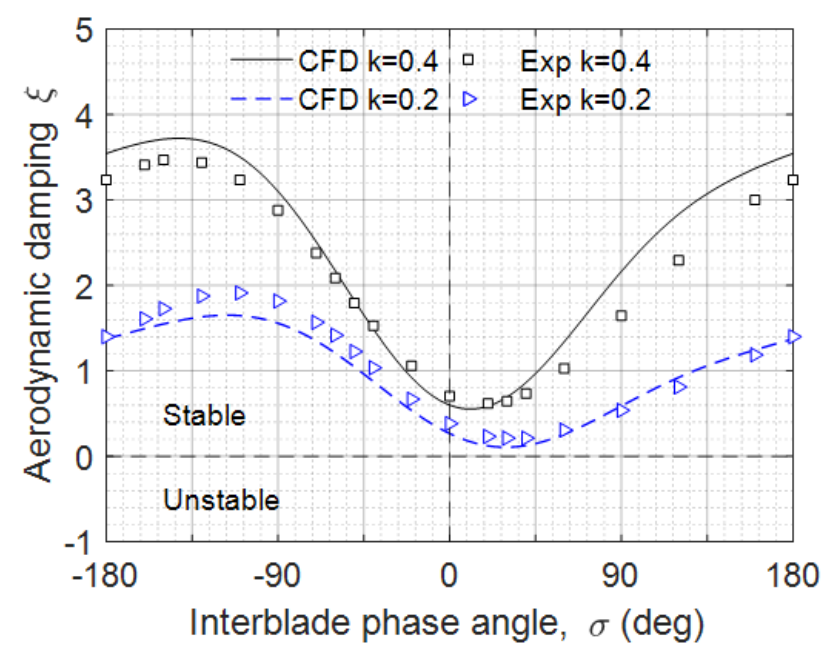

Fig. 2 Overall damping for bending mode at $k=0.2$ and 0.4

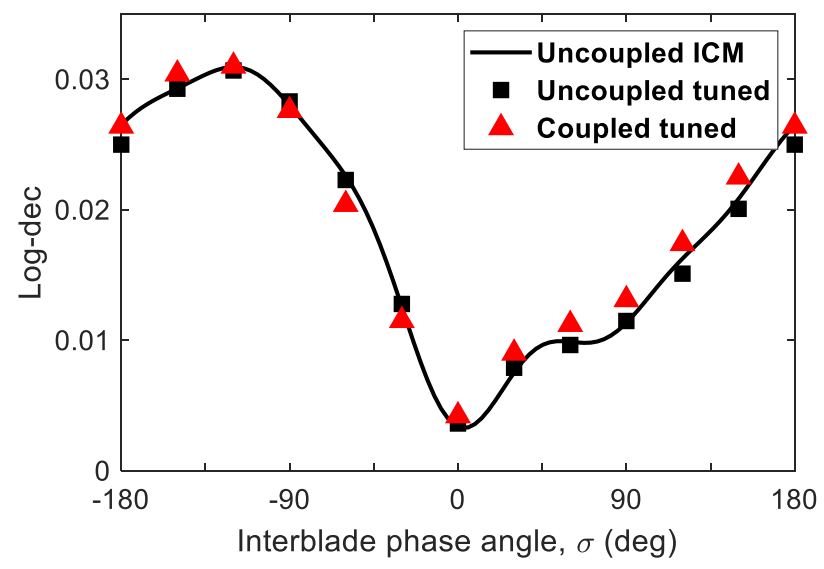

Fig. 3 Overall damping for torsion mode at $k=0.8$ 
ASME Journal of Engineering for Gas Turbines and Power

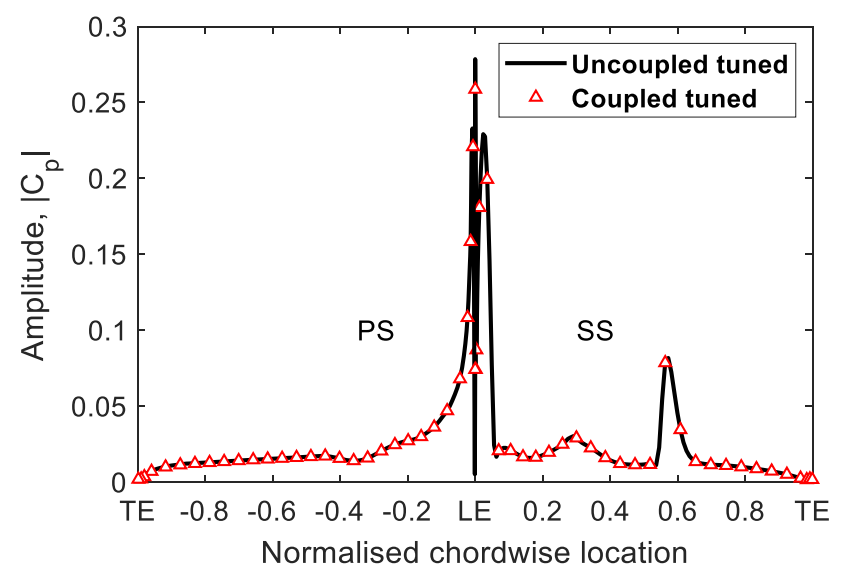

(a)

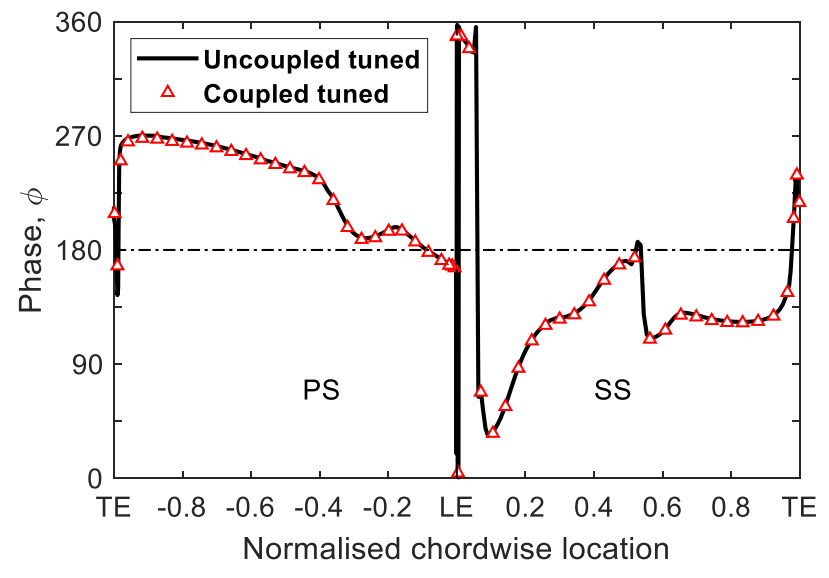

(b)

Fig. 4 Unsteady pressure responses at IBPA $\sigma=0^{0}$ : (a) Amplitude and b) Phase angle 
ASME Journal of Engineering for Gas Turbines and Power

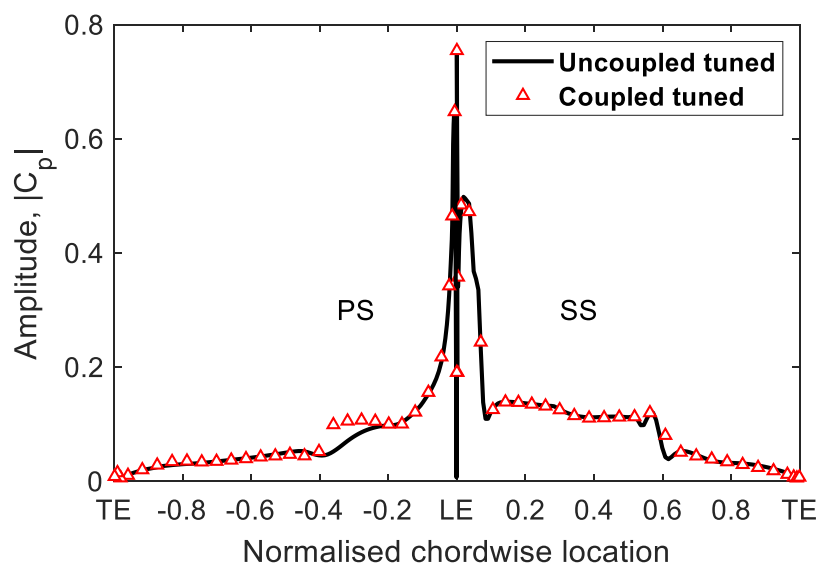

(a)

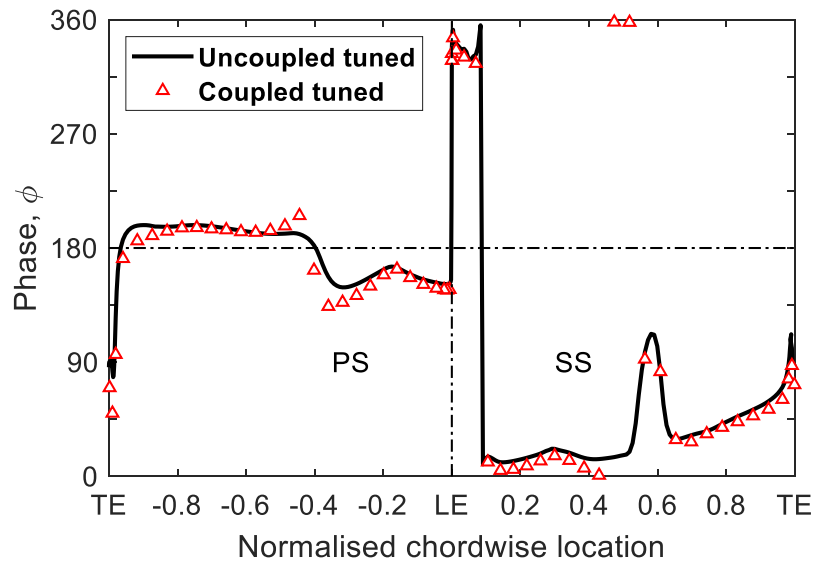

(b)

Fig. 5 Unsteady pressure responses at IBPA $\sigma=-120^{\circ}$ : (a) Amplitude and b) Phase angle 


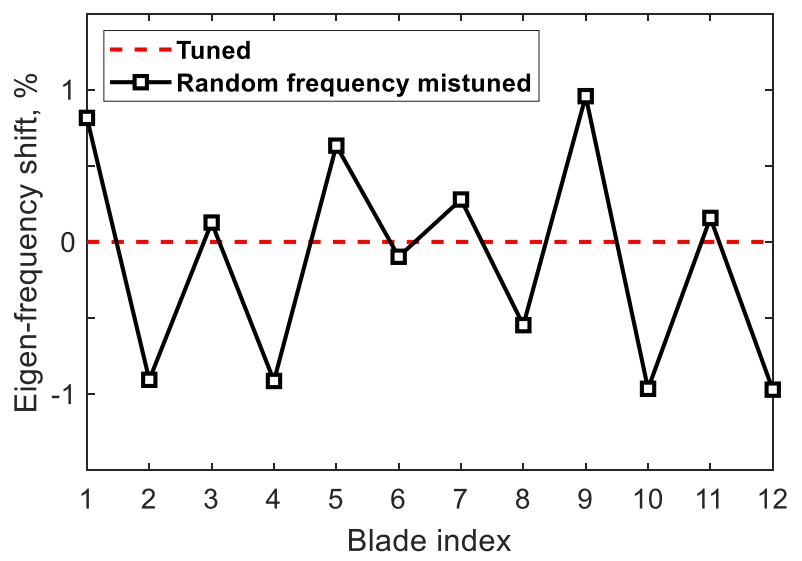

Fig. 6 Random mistuning pattern

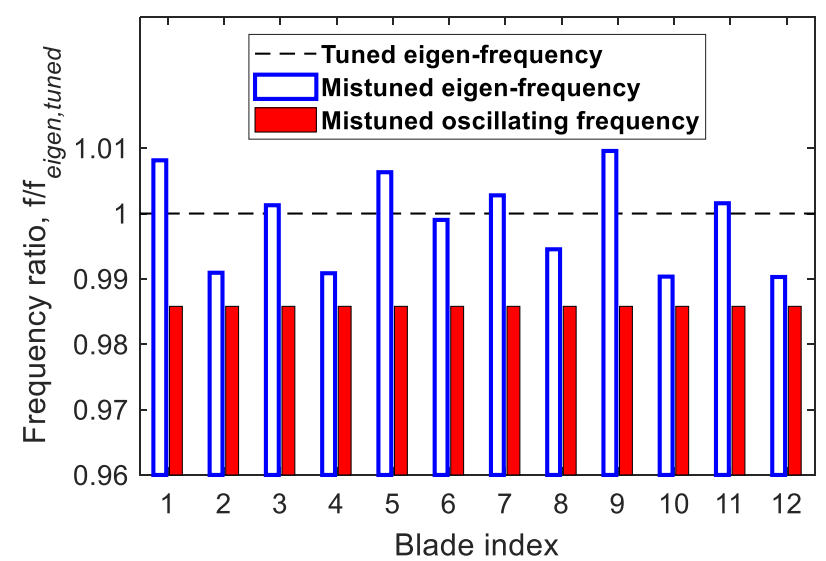

Fig. 7 Random structural mistuning frequency distribution for all blades 


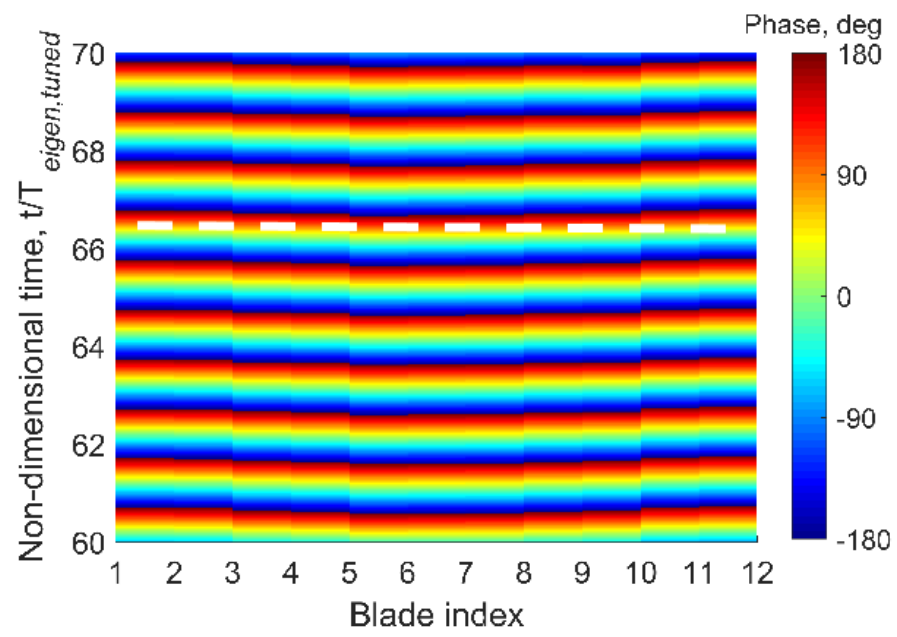

Fig. 8 Random structural mistuning phase distribution for all blades

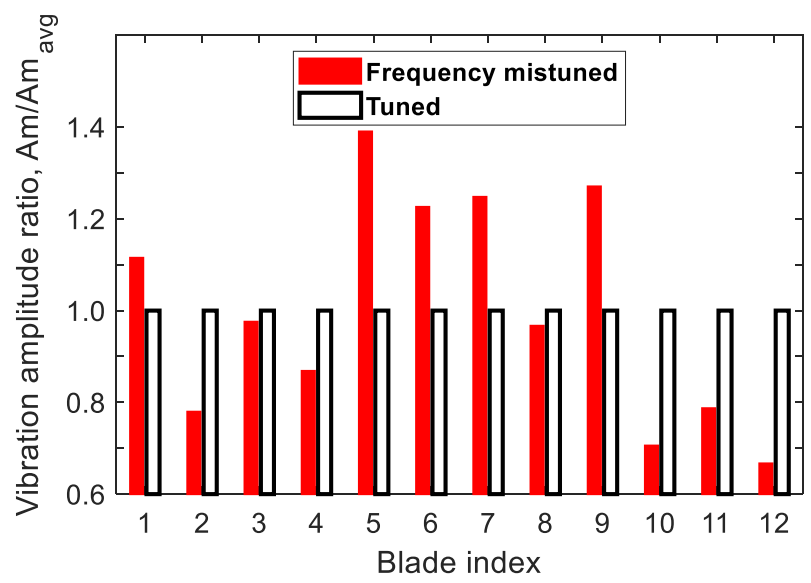

Fig. 9 Random structural mistuning amplitude distribution for all blades 


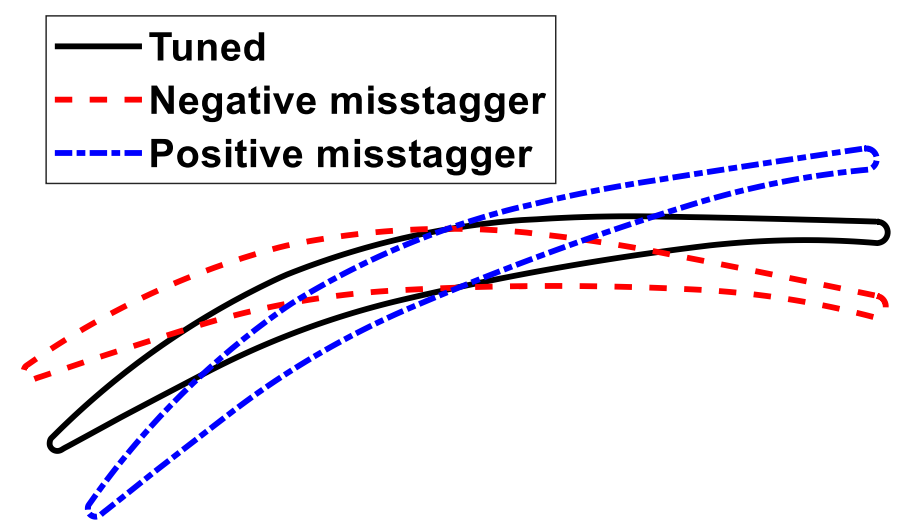

Fig. 10 Aerodynamic mistuning illustration

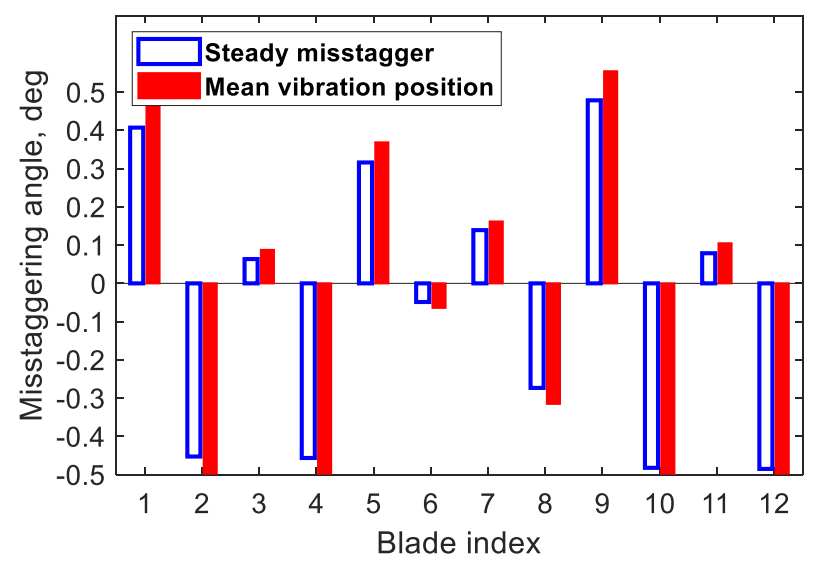

Fig. 11 Steady/mean mis-staggering for all blades in the random aerodynamic mistuning 


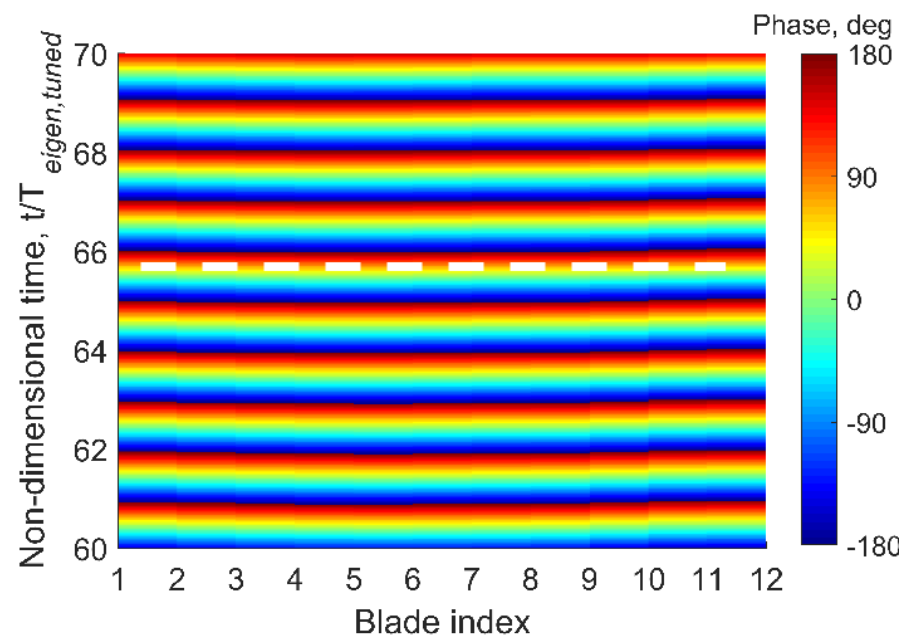

Fig. 12 Random aerodynamic mistuning phase distribution for all blades

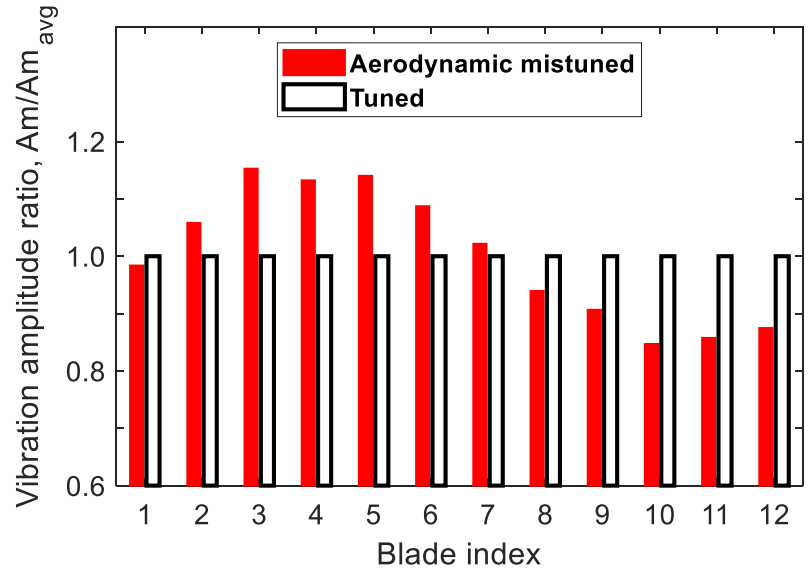

Fig. 13 Random aerodynamic mistuning amplitude distribution for all blades 


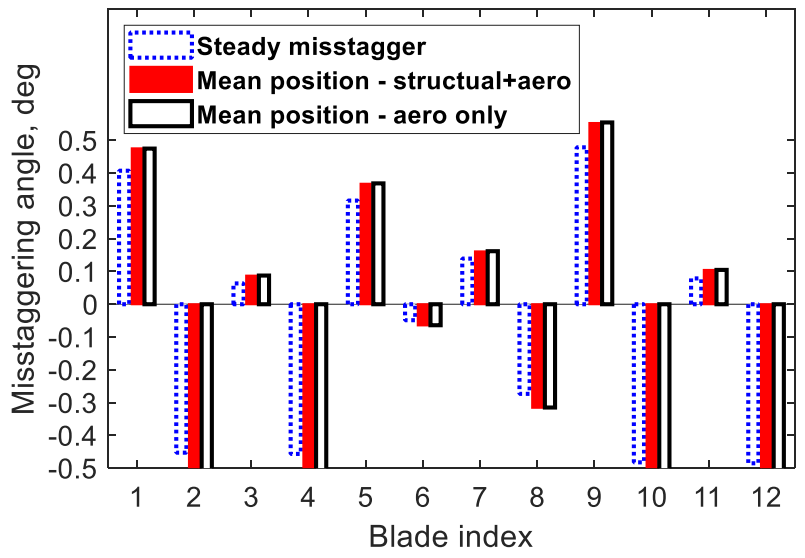

Fig. 14 Steady/mean mis-staggering for all blades in the concurrent structural-

aerodynamic mistuning

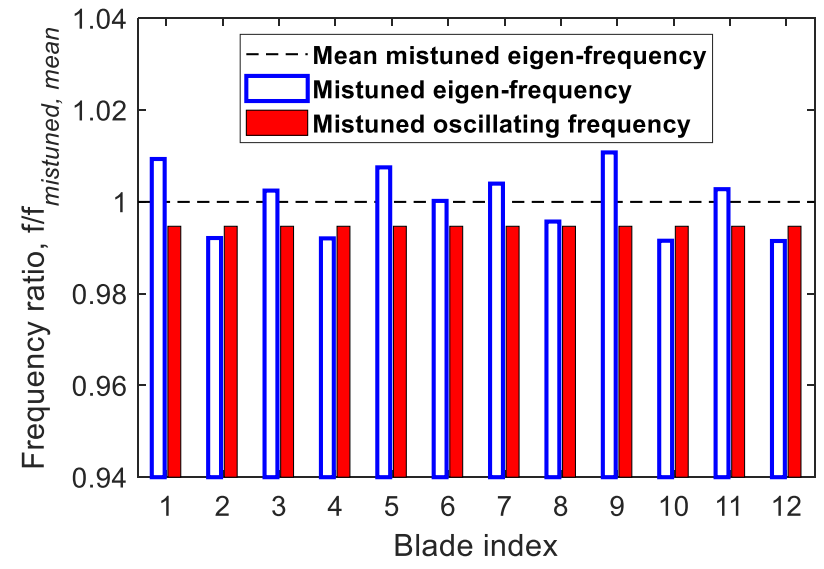

Fig. 15 Concurrent structural-aerodynamic mistuning frequency distribution for all

blades 
ASME Journal of Engineering for Gas Turbines and Power

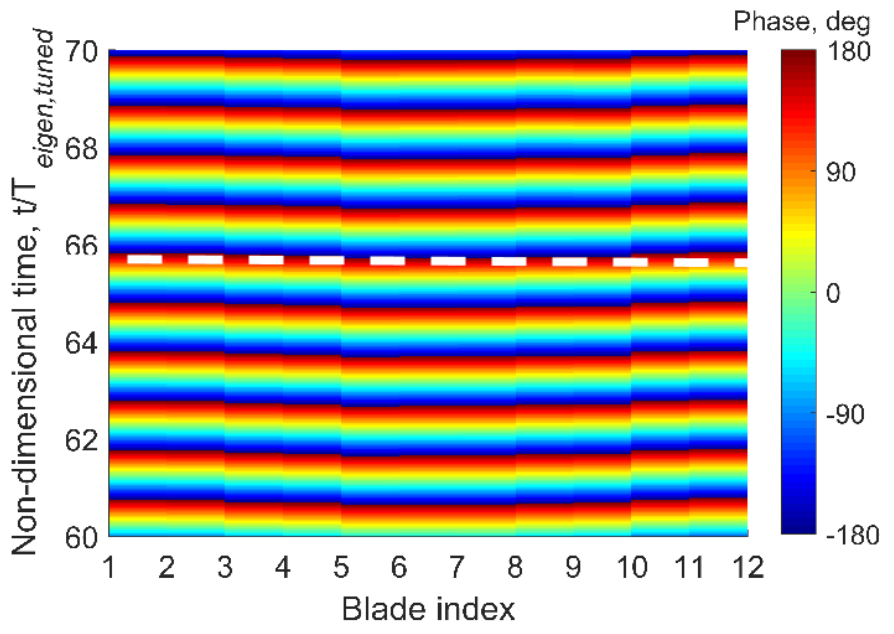

Fig. 16 Concurrent structural-aerodynamic mistuning phase distribution for all blades

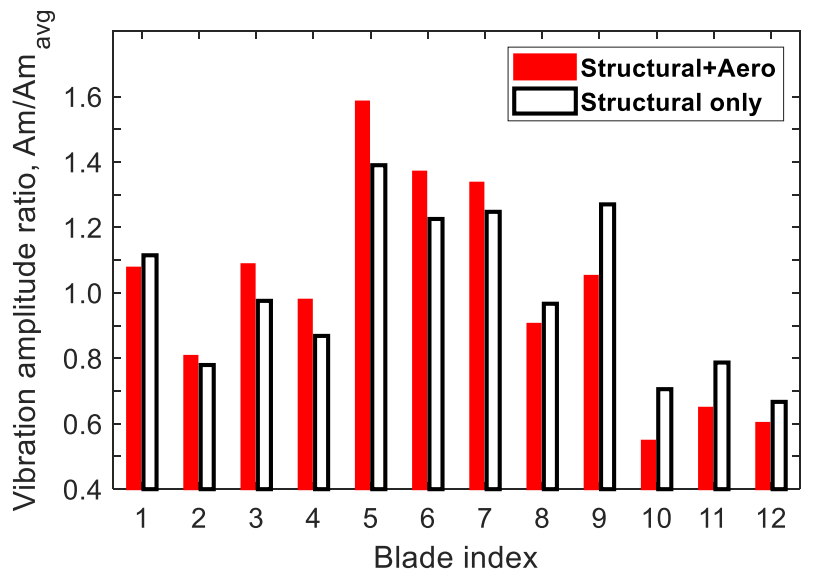

Fig. 17 Concurrent structural-aerodynamic mistuning amplitude distribution for all blades 


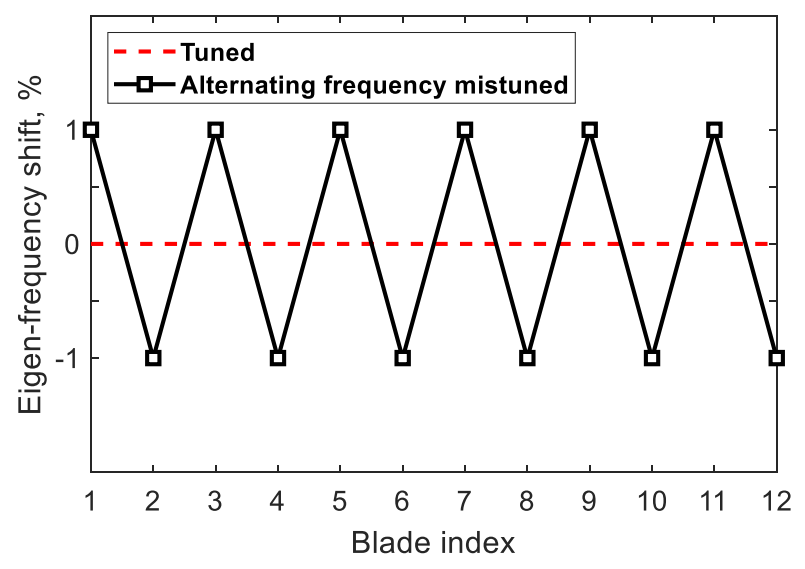

Fig. 18 Alternating mistuning pattern of $1 \%$

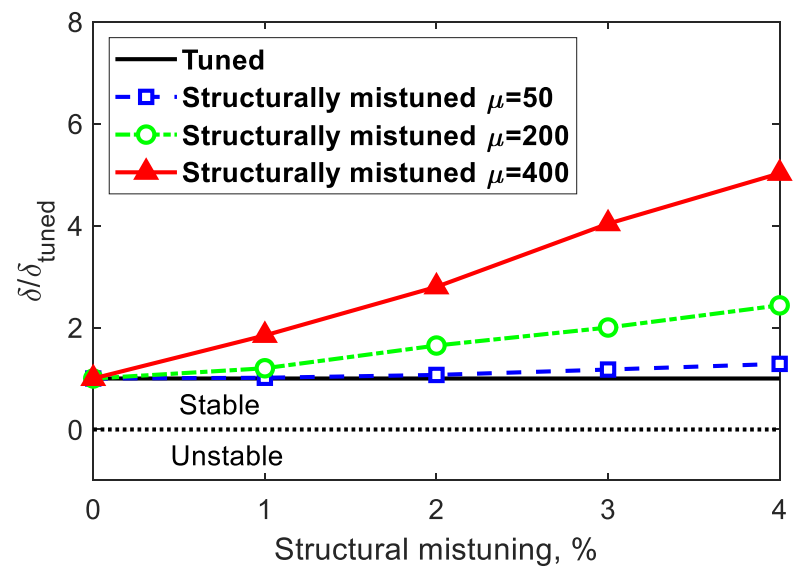

Fig. 19 Alternating structural mistuning $\log$-dec at $k=0.8$ 
ASME Journal of Engineering for Gas Turbines and Power

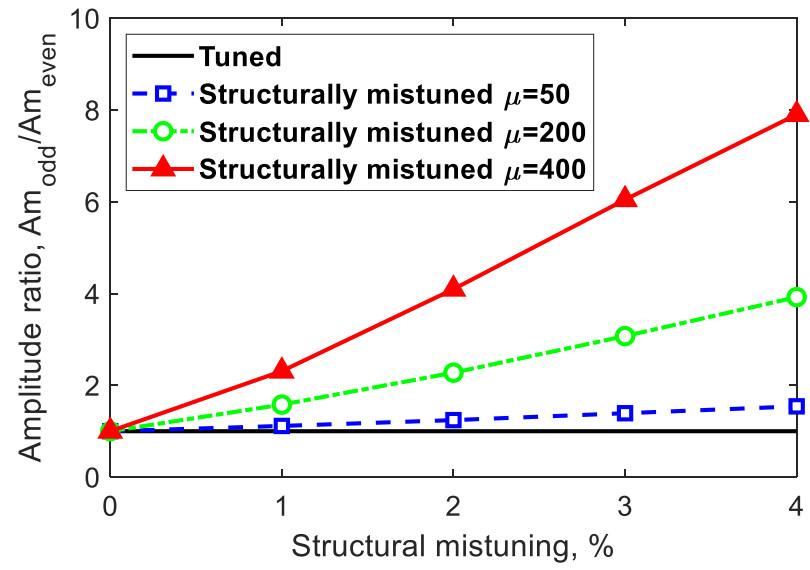

Fig. 20 Alternating structural mistuning amplitude ratio at $\mathrm{k}=0.8$

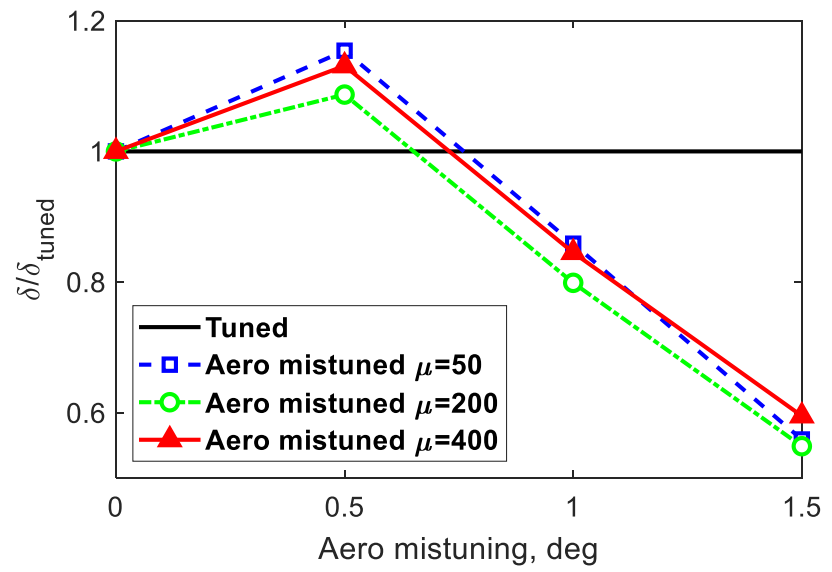

Fig. 21 Alternating aerodynamic mistuning log-dec at $k=0.8$ 
ASME Journal of Engineering for Gas Turbines and Power

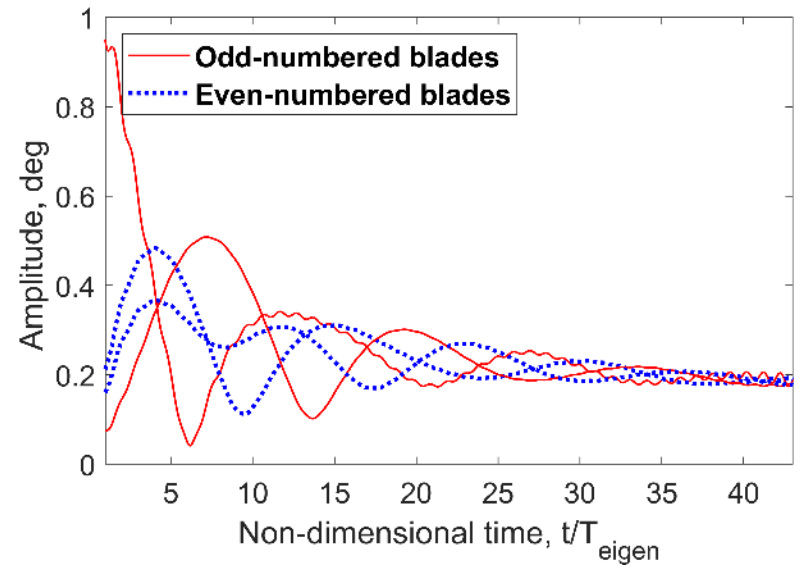

(a)

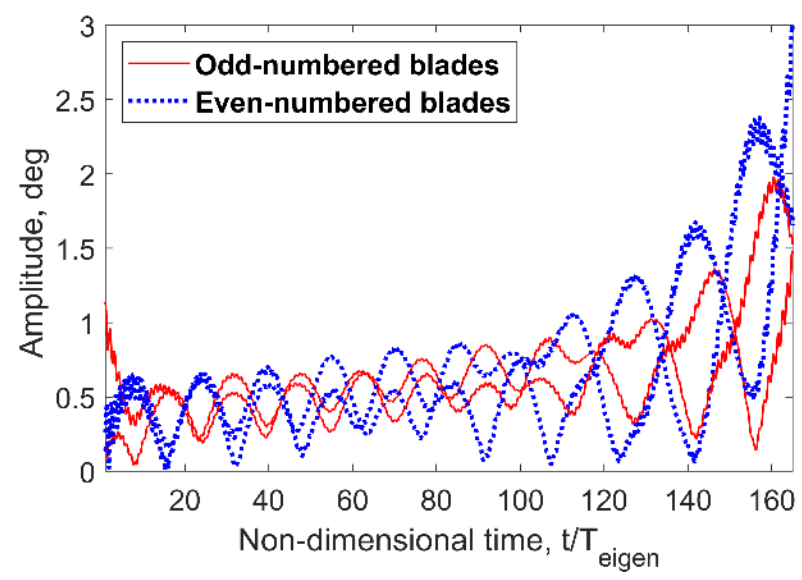

(b)

Fig. 22 Amplitude evolution for the: (a) Aerodynamically tuned + Structurally tuned and (b) Alternating aero-mistuning $1.5 \mathrm{deg}+$ Structurally tuned cascade 
ASME Journal of Engineering for Gas Turbines and Power

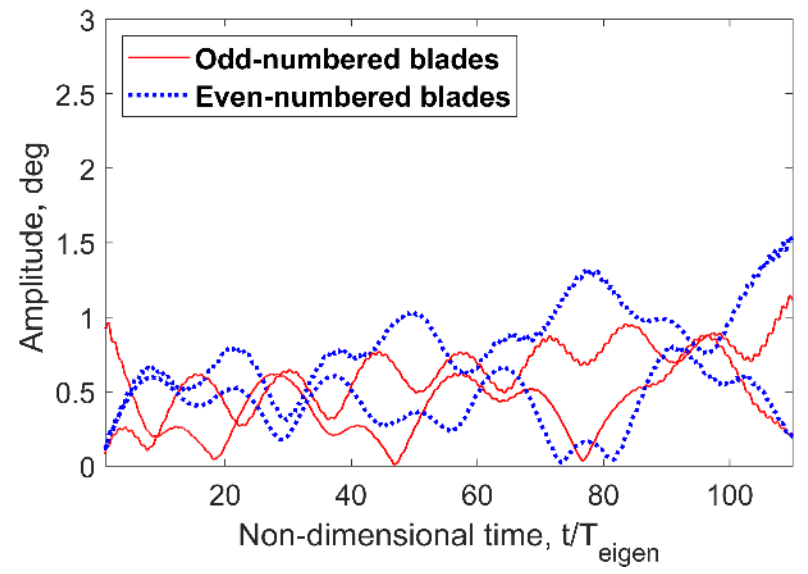

(a)

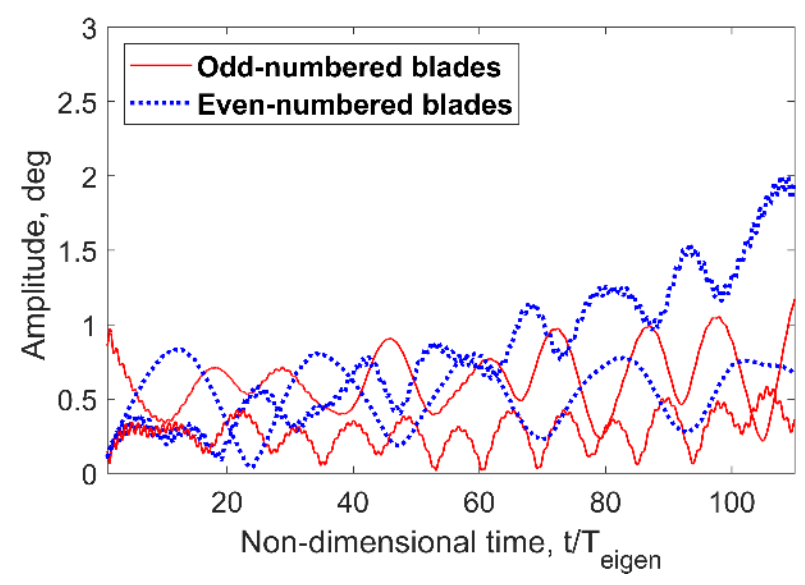

(b)

Fig. 23 Amplitude evolution for the: (a) Alternating aero-mistuning 1.5deg + Alternating structural mistuning $1 \%$ and (b) Alternating aero-mistuning 1.5deg + Alternating structurally mistuning $4 \%$ cascade 
ASME Journal of Engineering for Gas Turbines and Power

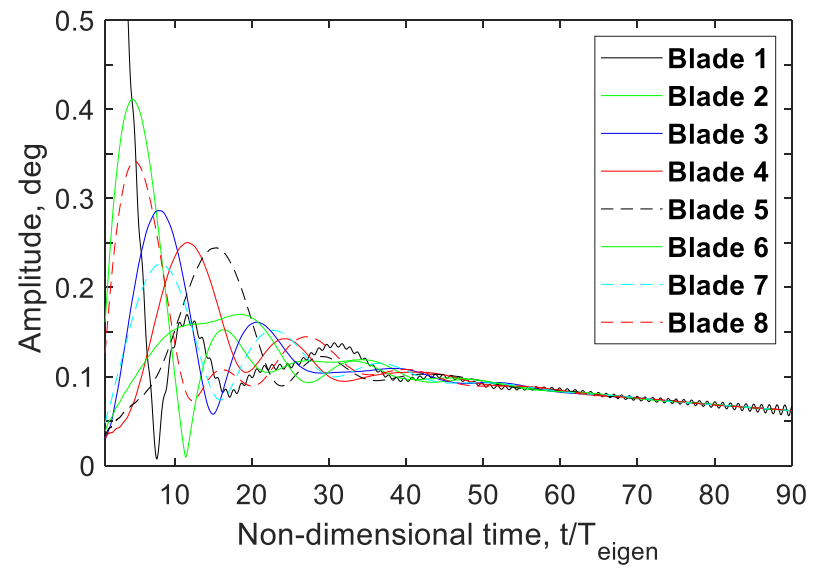

(a)

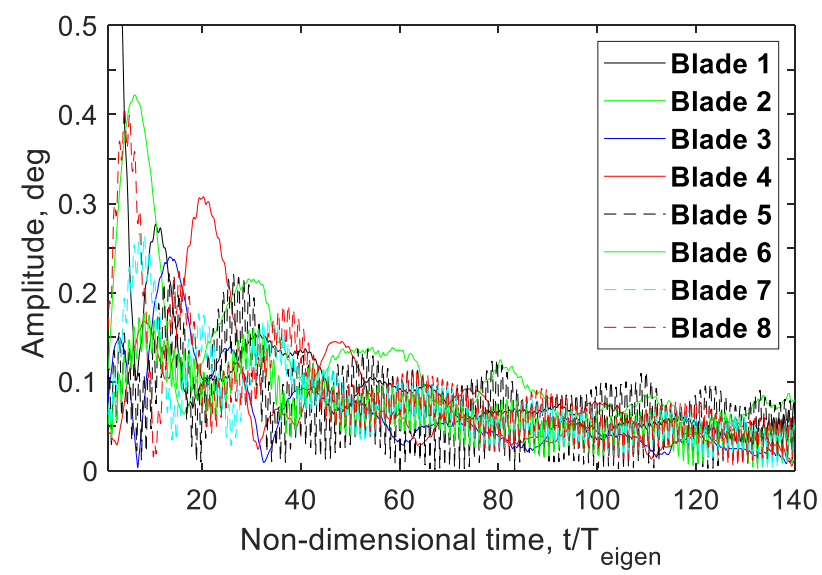

(b)

Fig. 24 Amplitude evolution for the: (a) Aerodynamically tuned + Structurally tuned and

(b) Random aero-mistuning $1.5 \mathrm{deg}+$ Structurally tuned cascade 
ASME Journal of Engineering for Gas Turbines and Power

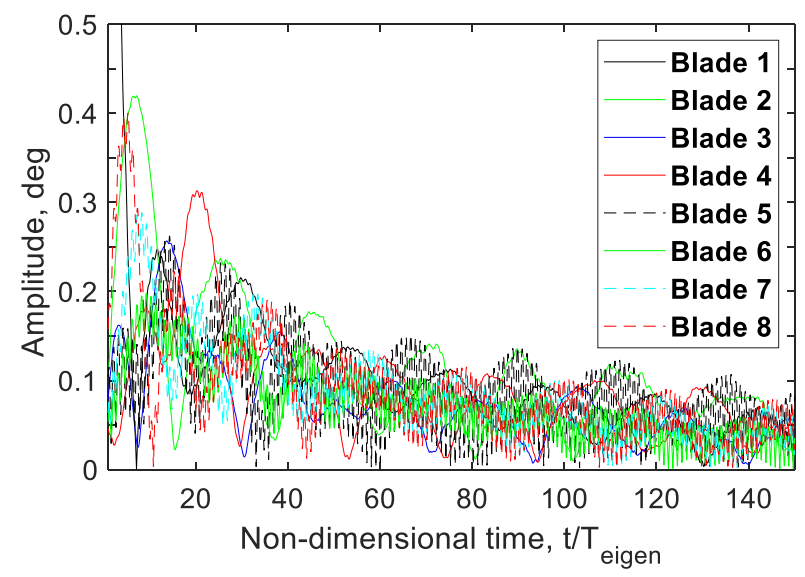

(a)

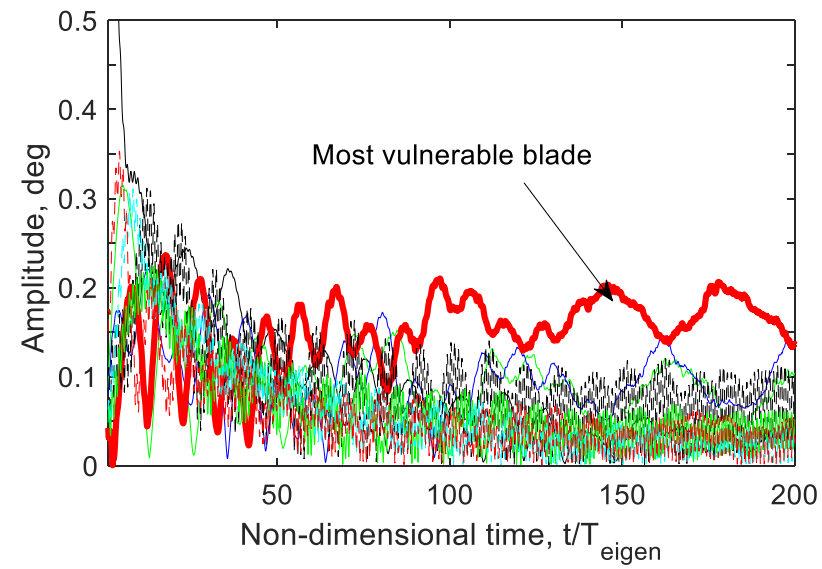

(b)

Fig. 25 Amplitude evolution for the: (a) Random aero-mistuning 1.5deg + Random structural mistuning $1 \%$ and (b) Random aero-mistuning 1.5deg + Random structurally mistuning $4 \%$ cascade 
ASME Journal of Engineering for Gas Turbines and Power

\section{Table Caption List}

Table 1 Experimental and Numerical Operating Conditions

Phan, GTP-21-1518 
ASME Journal of Engineering for Gas Turbines and Power

Table 1 Experimental and Numerical Operating Conditions

Conditions Experimental Numerical

$R e$ (based on blade chord and exit velocity) $\times 10^{5}$

$1.95 \quad 1.967$

Exit isentropic velocity $V_{\text {ref }}, \mathrm{ms}^{-1}$

19.5

20.4 